\title{
Improving the Efficiency of Control Signaling in Wireless Multiple Access Systems
}

\author{
Reza Moosavi
}

\section{SOMMUNICATION SŶstems}

Division of Communication Systems Department of Electrical Engineering (ISY)

Linköping University, SE-581 83 Linköping, Sweden www.commsys.isy.liu.se

Linköping 2013 
Improving the Efficiency of Control Signaling in Wireless Multiple Access Systems

(c) 2013 Reza Moosavi, unless otherwise noted.

ISBN: 978-91-7519-477-6

ISSN 0345-7524

Printed in Sweden by LiU-Tryck, Linköping 2013 
To my lovely wife, my beloved parents, and my dear sisters; without them I would not be here. 

"Even if you are on the right track, you'll get run over if you just sit there." Will Rogers 



\section{Abstract}

Prior to the transmission of payload data in any multiple access system, there is generally a need to send control information such as scheduling assignments, transmission parameters and HARQ acknowledgments. This process is called control signaling and has a significant impact on the overall system performance. This dissertation considers different aspects of control signaling and proposes some novel schemes for improving it. The dissertation is split into two parts where in the first part the focus is on the transmission of scheduling assignments, and in the second part the focus is on improving the "blind decoding" process that is used to achieve adaptive coding and modulation in transmission of control information.

More specifically, in the first part of the dissertation we first compare the two conventional schemes for control signaling using extensive system simulations. In doing so, we use practical assumptions on the scheduling algorithm as well as on the compression and transmission of the scheduling information. We then provide two schemes for reducing the amount of control signaling that concerns the transmission of scheduling assignments. The first scheme, which is reminiscent of source coding with side information, uses the knowledge that each user has about its own channel condition to compress the scheduling information more effectively. The second scheme uses the fact that in wireless multiple access systems, a user with a given channel condition can in principle decode the data intended to the users that have weaker channels. Therefore, the idea is to send the scheduling information of different terminals in a differential manner starting from the user with the weakest channel and letting all the terminals overhear the transmission of one another. Finally, in the last section of this part we use some of the recent results in information theory to form a general framework for the comparison of different control signaling schemes. We formulate an optimization problem that for a given desired error probability finds the minimum required number of channel uses for a given signaling scheme.

In the second part of the thesis, we propose three schemes for reducing the complexity of the blind decoding process. The first one is a novel scheme for fast blind identification of channel codes. More precisely, we propose an efficient algorithm that for a given sequence of received symbols and a given linear channel code, finds the posterior probability that all the parity check relations of the code are satisfied. We then use this quantity to perform a sequential statistical hypotheses test that 
reduces the computational complexity of blind decoding. The idea in the second scheme is to broadcast a control message prior to the transmission of control information to instruct only a subset of the terminals (ideally only those terminals that have been scheduled for reception of payload data and hence benefit from performing a blind search attempt) to perform blind search decoding, which can be used for instance in LTE to reduce the complexity of the blind decoding process. Finally, in the third scheme we propose to split the CRC, used by the terminals to find their control information, into two parts and inject one part early in the control data stream so that the terminals can detect early if the current decoding attempt will be successful, which ultimately reduces the blind decoding complexity. 


\section{Populärvetenskaplig Sammanfattning}

Ända från början har den trådlösa kommunikationen förändrat livet för många människor världen över. För närvarande använder mer än hälften av världens befolkning dagligen trådlösa enheter för olika ändamål. De tidiga trådlösa systemen kunde erbjuda enkla och specifika lågtaktstjänster. Dagens system kan dock stödja en mängd mer avancerade tjänster som kräver kommunikation med hög datahastighet. Exempel på sådana tjänster är webb-surfning och strömmade multimediaprogram. För att möta de höga kraven som ställs på dagens system har många tekniska lösningar föreslagits. Många av dessa lösningar är kraftfulla i den mening att de ökar systemets prestanda. Å andra sidan inför de en betydande styrtrafiksoverhead på systemet. Kontrollsignalering innebär att skicka styrinformation som är nödvändig för att upprätta och/eller upprätthålla anslutningen. Detta är tillägg till den nyttoinformation som överförs under förbindelsen.

Styrsignalering förbrukar delar av radioresurserna som annars kan användas för överföring av nyttolastdata. Därför är det viktigt att förbättra effektiviteten i överföringen av kontrollinformationen. Denna avhandling betraktar olika aspekter av styrsignalering och föreslår några nya lösningar för att förbättra den.

Avhandlingen är uppdelad i två delar. I den första delen fokuserar vi på överföring av schemaläggningsuppdrag som beskriver var i tid/frekvensdomänen nyttolastdata för olika användare är belägen. Vi jämför först konventionella metoder för att skicka schemaläggningsuppdrag. Vi föreslår därefter två nya metoder som kräver betydligt mindre andel radioresurser jämfört med de konventionella metoderna.

I den andra delen ligger fokus på att förbättra "blindavkodningsprocessen" som används för överföring av styrinformation i kommande trådlösa system (kallas 4G eller LTE). I synnerhet, föreslår vi tre lösningar för att minska komplexiteten i den blindavkodningsprocessen. 


\section{Acknowledgments}

First and foremost, I would like to thank my supervisor Prof. Erik G. Larsson for offering me a research carrier and for all his valuable support that guided me in the right direction. Secondly, I would like to express my deepest gratitude to Dr. Jonas Eriksson for his guidance, help and being open to any brainstorming discussions, and to Prof. Danyo Danev, my co-supervisor, for helping me with my research and also in proofreading the thesis. Also, I would like to thank my research sponsors and my current colleagues at Ericsson Research Center at Linköping, specially Dr. Gunnar Bark for offering me another research carrier, Dr. Niclas Wiberg and Dr. Pål Frenger for their close collaborations.

Many thanks to my colleagues (and former colleagues) at the Communication Systems division and our neighboring research group Information Coding division. Specially, I would like to express my appreciation towards Prof. Mikael Olofsson and Prof. Lasse Alfredsson for their guidance in teaching related matters, to Dr. Eleftherios Karipidis, Dr. Ebrahim Avazkonandeh Gharavol, Dr. Saif K. Mohammed and Dr. Daniel Persson for their help and research discussions. I would also like to thank the PhD students (and former PhD students) at the Communication Systems division, specially to my roommate T. V. K. Chaitanya for always listening to my immature ideas, Mirsad, Hien and Antonis for the interesting discussions and to the rest for making such an amazing atmosphere in the group.

Finally, I would like to thank all the people that somehow made me choose the research carrier. This includes many teachers and professors in my home city Isfahan, during my master study at Chalmers University of Technology and at Swiss Federal Institute of Technology Zurich (ETHZ). Many thanks should be devoted to my family and friends for always believing in me and for their supports. Especially, I would like to express my deepest gratitude to my mom and dad, from whom I have every thing in my life, to my sisters Zari and Samira and their families, to my parent-in-law, and to my brother-in-low Forood. Last but not least, I would like to thank my wife Tahmineh for her support, patience and kindness. Thank you Tahmineh for all the meaning and all the fortune that you brought to my life.

Linköping, December 2013

Reza Moosavi 


\section{Abbreviations}

$3 \mathrm{G}$

3GPP

$4 \mathrm{G}$

ACK

AMC

ARQ

AWGN

BER

BLER

BPSK

BSC

CCE

$\mathrm{CDF}$

CDMA

CLT

CQI

CRC

CSI

DCET

DCI

EB

FLOPS
Third Generation

Third Generation Partnership Project

Forth Generation

Acknowledgment

Adaptive Modulation and Coding

Automatic Repeat Request

Additive White Gaussian Noise

Bit Error Rate

Block Error Rate

Binary Phase Shift Keying

Binary Symmetric Channel

Control Channel Element

Cumulative Distribution Function

Code Division Multiple Access

Central Limit Theorem

Channel-Quality Indicator

Cyclic Redundancy Check

Channel State Information

Differential Compression Encoding and Transmission

Downlink Control Information

Exabyte

FLoating-point Operations Per Second 


\begin{tabular}{|c|c|}
\hline HARQ & Hybrid Automatic Repeat Request \\
\hline HSDPA & High Speed Downlink Packet Access \\
\hline HSPA & High Speed Packet Access \\
\hline HSUPA & High Speed Uplink Packet Access \\
\hline ICC & Inter-Cell Coordinator \\
\hline i.i.d. & Independent and Identically Distributed \\
\hline ILS & Iterative Local Search \\
\hline i.n.d. & Independent and Non-identically Distributed \\
\hline ITU & International Telecommunication Union \\
\hline JCEB & Joint Compression Encoding and Broadcast \\
\hline LDPC & Low-Density Parity-Check \\
\hline LLR & Log-Likelihood Ratio \\
\hline LTE & Long Term Evolution \\
\hline MIMO & Multiple-Input Multiple-Output \\
\hline ML & Maximum Likelihood \\
\hline NACK & Negative Acknowledgment \\
\hline OFDM & Orthogonal Frequency Division Multiplexing \\
\hline OFDMA & Orthogonal Frequency Division Multiple Access \\
\hline PAM & Pulse Amplitude Modulation \\
\hline $\mathrm{PCFICH}$ & Physical Control Format Indicator Channel \\
\hline $\mathrm{PDCCH}$ & Physical Downlink Control Channel \\
\hline PDF & Probability Distribution Function \\
\hline PDP & Please-Decode-Blindly \\
\hline $\mathrm{PHICH}$ & Physical HARQ Indicator Channel \\
\hline $\mathrm{PUCCH}$ & Physical Uplink Control Channel \\
\hline QoS & Quality of Service \\
\hline QPSK & Quadrature Phase Shift Keying \\
\hline SCET & Separate Compression Encoding and Transmission \\
\hline SINR & Signal to Interference plus Noise Power Ratio \\
\hline SNR & Signal to Noise Power Ratio \\
\hline SPP & Syndrome Posterior Probability \\
\hline SPRT & Sequential Probability Ratio Test \\
\hline SSHT & Sequential Statistical Hypothesis Test \\
\hline TTI & Transmission Time Interval \\
\hline UVLC & Universal Variable Length Code \\
\hline VoIP & Voice over Internet Protocol \\
\hline WCDMA & Wideband Code Division Multiple Access \\
\hline WiMAX & Worldwide Interoperability for Microwave Access \\
\hline WINNER & Wireless World Initiative New Radio \\
\hline
\end{tabular}




\section{Contents}

Abstract vii

Populärvetenskaplig Sammanfattning (in Swedish) ix

Acknowledgments $\quad$ xi

Abbreviations $\quad$ xiii

I Introduction 1

1 Background 3

1.1 Main Techniques to Achieve Higher Data Rates . . . . . . . . . . . . 3

1.1.1 Adaptive Modulation and Coding . . . . . . . . . . . . . 4

1.1.2 Channel Dependent Scheduling . . . . . . . . . . . . . . . 4

1.1.3 Hybrid Automatic Repeat Request . . . . . . . . . . . . . 5

1.1.4 Multiple Input Multiple Output (MIMO) . . . . . . . . . . . 5

1.2 Control Signaling . . . . . . . . . . . . . . . 5

1.3 Thesis Overview ...................... 6

2 Control Signaling in Wireless Multiple Access Systems $\quad 7$

2.1 High Speed Packet Access (HSPA) . . . . . . . . . . . . . . 7

2.2 A Brief Overview of LTE Downlink . . . . . . . . . . . . . 8

2.2.1 Channel Dependent Scheduling and Rate Adaptation . . . . 9

2.2.2 HARQ with Soft Combining . . . . . . . . . . . . . . . 9 9

2.2.3 Support for Multiple Antennas . . . . . . . . . . . . . . . 10

2.3 The Control Channel in LTE Downlink . . . . . . . . . . . . . . . . 10

2.3.1 Physical Control Format Indicator Channel . . . . . . . . . . 11

2.3.2 Physical HARQ Indicator Channel . . . . . . . . . . . . . . . 12

2.3.3 Physical Downlink Control Channel . . . . . . . . . . . . . 12

2.3.4 Blind Decoding of PDCCH:s . . . . . . . . . . . 15

3 Literature Overview on Control Signaling $\quad 17$

3.1 Research Related to Signaling Overhead . . . . . . . . . . . . . . . . 17

3.2 Contributions of the Dissertation . . . . . . . . . . . . . . 19

4 Summary of Specific Contributions of the Dissertation 23

4.1 Included Papers . . . . . . . . . . . . . . . . . . . . . . . . . 23

4.2 Not Included Papers . . . . . . . . . . . . . . . . . . . . . 27

5 Conclusions and Future Research Directions 29 
5.1 Conclusions . . . . . . . . . . . . . . . . . . . . . 29

5.2 Future Research Directions . . . . . . . . . . . . . . . . . . 31

\section{Transmission of Scheduling Assignments}

A Comparison of Strategies for Signaling of Scheduling Assignments in Wireless OFDMA

1 Introduction . . . . . . . . . . . . . . . . . 42

1.1 Background and Motivation . . . . . . . . . . . . . . . . 42

1.2 Related Work and Contributions . . . . . . . . . . . . . . . 43

2 System Model and Preliminaries . . . . . . . . . . . . . . . . . . . . 44

3 Scheduling Granularity . . . . . . . . . . . . . . . . . . . . . . . . 47

4 Scheduling Strategies . . . . . . . . . . . . . . . . . . . 48

4.1 System-Throughput Maximizing Scheduler . . . . . . . . . 49

4.2 Round-Robin Scheduler . . . . . . . . . . . . . . . . . 49

4.3 Proportional Fair Scheduler . . . . . . . . . . . . . 50

5 Signaling of the Scheduling Assignments . . . . . . . . . . . . . 50

$5.1 \quad$ Compression of Scheduling Maps . . . . . . . . . . . . . 51

5.2 Model for Transmission of the Compressed Scheduling Information . . . . . . . . . . . . . . . 52

5.3 Joint Compression, Encoding and Broadcast (JCEB) Scenario 54

5.4 Separate Compression, Encoding and Transmission (SCET) Scenario . . . . . . . . . . . . . . 56

5.5 Remark on Error Probabilities of the Scheduling Information 58

6 System Simulation Model _. . . . . . . . . . . . . . . . . . . . 59

$7 \quad$ Numerical Results . . . . . . . . . . . . . . . . . . . . . . . . 60

$7.1 \quad$ Signaling Overhead Ratio . . . . . . . . . . . . . . . 61

$7.2 \quad$ Spectral Efficiency . . . . . . . . . . . . . . . 63

8 Conclusions . . . . . . . . . . . . . . . . . . 64

B Reducing Physical Layer Control Signaling Using Mobile$\begin{array}{ll}\text { Assisted Scheduling } & \mathbf{7 7}\end{array}$

1 Introduction . . . . . . . . . . . . . . . . . . . . . 80

2 System Model . . . . . . . . . . . . . . . . . . . . . . . 81

3 Conventional Approach for Signaling of Scheduling Assignments . . 83

4 Proposed Mobile-Assisted Scheduling Scheme . . . . . . . . . . . . . 84

5 Theoretical Justification of the Proposed Scheme . . . . . . . . . . . 87

$5.1 \quad$ Case 1: i.i.d. scheduling metrics . . . . . . . . . . . . 89

5.2 Case 2: i.n.d. scheduling metrics . . . . . . . . . . . 89

$6 \quad$ Optimal Scheduling to Minimize Signaling Overhead . . . . . . . . . 91

6.1 Optimum Scheduling Assignment According to the Conventional Scheme . . . . . . . . . . . . . . . . . 91

6.2 Optimum Scheduling Assignment According to MobileAssisted Scheduling Scheme . . . . . . . . . . . . . . . 94

6.3 Efficient Algorithm to Solve The First Sub-Problem . . . . . 97

$7 \quad$ Numerical Examples . . . . . . . . . . . . . . . . . . . . . . . . 99 
Future Work and Conclusion . . . . . . . . . . . . . . . . 104

C Differential Signaling of Scheduling Information in Wireless Mul$\begin{array}{lr}\text { tiple Access Systems } & 107\end{array}$

1 Introduction . . . . . . . . . . . . . . . . . . . . . . . 110

$1.1 \quad$ Background and Motivation . . . . . . . . . . . . 110

1.2 Related Work . . . . . . . . . . . . . . . . . . . . 110

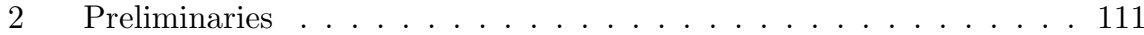

3 Proposed Approach . . . . . . . . . . . . . . . . . . . . . . . 112

4 Theoretical Justification of the Proposed Scheme . . . . . . . . . . . 115

5 Simulation Model . . . . . . . . . . . . . . . . . . . 117

6 Simulation results . . . . . . . . . . . . . . . . . . . . . 119

7 Conclusion . . . . . . . . . . . . . . . . 120

D Optimized Encoding of Scheduling Assignments Using Finite $\begin{array}{lr}\text { Blocklength Coding Bounds } & 125\end{array}$

1 Introduction . . . . . . . . . . . . . . . . . . . . . . 128

2 System Model . . . . . . . . . . . . . . . . . . . . . . . 128

3 Performance Comparison of Signaling Schemes . . . . . . . . . . . 130

3.1 Compression of the Scheduling Maps . . . . . . . . . . . . . . 130

3.2 Transmission of Scheduling Assignments . . . . . . . . . . . . 131

4 Optimization of the Number of Resources . . . . . . . . . . . . . . . 132

5 Numerical Comparison . . . . . . . . . . . . . . . . . . . 134

6 Conclusion . . . . . . . . . . . . . . . . 136

III Blind Decoding Schemes 139

E Fast Blind Recognition of Channel Codes 141

1 Introduction . . . . . . . . . . . . . . . . . . . . . . . . . . 144

1.1 Contribution ...................... 145

2 Computing the Syndrome Posterior Probability . . . . . . . . . . . . 145

2.1 Computational Complexity of Computing SPP . . . . . . . . 147

2.2 Parity Check Matrices and the SPP . . . . . . . . . . . . 148

$2.3 \quad$ Approximation of the SPP . . . . . . . . . . . . 149

3 Using SPP for Blindly Identifying a Channel Code . . . . . . . . . . 149

3.1 Analysis of the Code Detection Performance . . . . . . . . . 151

3.2 Application of the Code Detection Scheme to Convolutional

Codes . . . . . . . . . . . . . . . . . . 155

4 Using SPP for Reducing The Computational Complexity of Blind

Decoding . . . . . . . . . . . . . . . . 156

$4.1 \quad$ Proposed Sequential Statistical Hypothesis Test . . . . . . . . 158

4.2 A Rule-of-Thumb for the Required Number of Observation . 160

5 Simulation Results . . . . . . . . . . . . . . . . . 162

6 Conclusions . . . . . . . . . . . . . . . . . . 166

A Computing the Mean and the Variance of $\gamma_{k} \ldots \ldots \ldots$

B Computing the Correlation Between $\gamma_{k}$ and $\gamma_{k^{\prime}} \ldots \ldots$. . . . . . . . 172 
F Fast Identification of Control Signaling Aided by Please-Decode$\begin{array}{lr}\text { Blindly (PDB) Messages } & 177\end{array}$

1 Introduction . . . . . . . . . . . . . . . . . . . . . 180

2 Related Work . . . . . . . . . . . . . . . . . . . . . 181

3 Proposed Scheme . . . . . . . . . . . . . . . . . . . . . . 182

4 Grouping Based on the Assignments History . . . . . . . . . . . . . . 183

5 Grouping Based on the Traffic Status . . . . . . . . . . . . . . . . 184

6 Theoretical Justification . . . . . . . . . . . . . . . . . . . . . 185

7 Numerical Illustration . . . . . . . . . . . . . . . . . . . . . . 187

8 Conclusion . . . . . . . . . . . . . . . . . 190

G Complexity Reduction of Blind Decoding Schemes Using CRC Splitting

1 Introduction . . . . . . . . . . . . . . . . . . 196

2 Related Work . . . . . . . . . . . . . . . . . 197

3 Contributions . . . . . . . . . . . . . . . . . . 197

4 Proposed Scheme: CRC Splitting . . . . . . . . . . . . . . . . 198

5 Split-CRC Error Detection Performance . . . . . . . . . . . . . . . . 199

$5.1 \quad$ Numerical Examples - Error Detection . . . . . . . . . . . . 201

6 Split-CRC Code Detection Performance . . . . . . . . . . . . . . . . 201

$6.1 \quad$ Numerical Examples - Code Detection . . . . . . . . . . . . 204

7 Complexity reduction . . . . . . . . . . . . . . 205

8 Conclusions . . . . . . . . . . . . . . . . . 207 


\section{Part I}

\section{Introduction}





\section{Chapter 1}

\section{Background}

During the 1890s, Guglielmo Marconi initiated the concept of using electromagnetic waves for communications which is now known as radio or wireless communications [1]. Half a century later, the first commercial wireless system was created in the United States, and since then many wireless systems have been introduced [2]. The early systems could provide some basic services to several individuals, whereas nowadays wireless systems provide more or less advanced services to more than half of the world's population, and this increase is predicted to be exponential in the coming years. In fact, the number of wireless-connected devices will exceed the world's population in 2014 [3]. This has an enormous impact on the data traffic capability of the wireless systems. This can be understood from Figure 1.1, where the average mobile traffic per month during the last two years is illustrated. Also in this figure, an estimate of the mobile traffic in the near future is given.

In order to meet these increasing demands on the data rate, many techniques have been designed, which resulted in an enormous evolution of the wireless systems in the last two decades. We start by briefly visiting the main techniques for achieving higher data rates in wireless systems.

\subsection{Main Techniques to Achieve Higher Data Rates}

Transmission over wireless channels is subject to errors. This is due to the fact that the strength of the received signal in wireless channels is varying with time and/or frequency. Hence, when the communication channel is in deep fading, that is when the communication channel does not have enough strength, it is very difficult to maintain a reliable data transmission. To combat the channel fading, new techniques have been found. The main techniques are as follows. 
$12 \mathrm{~EB}$
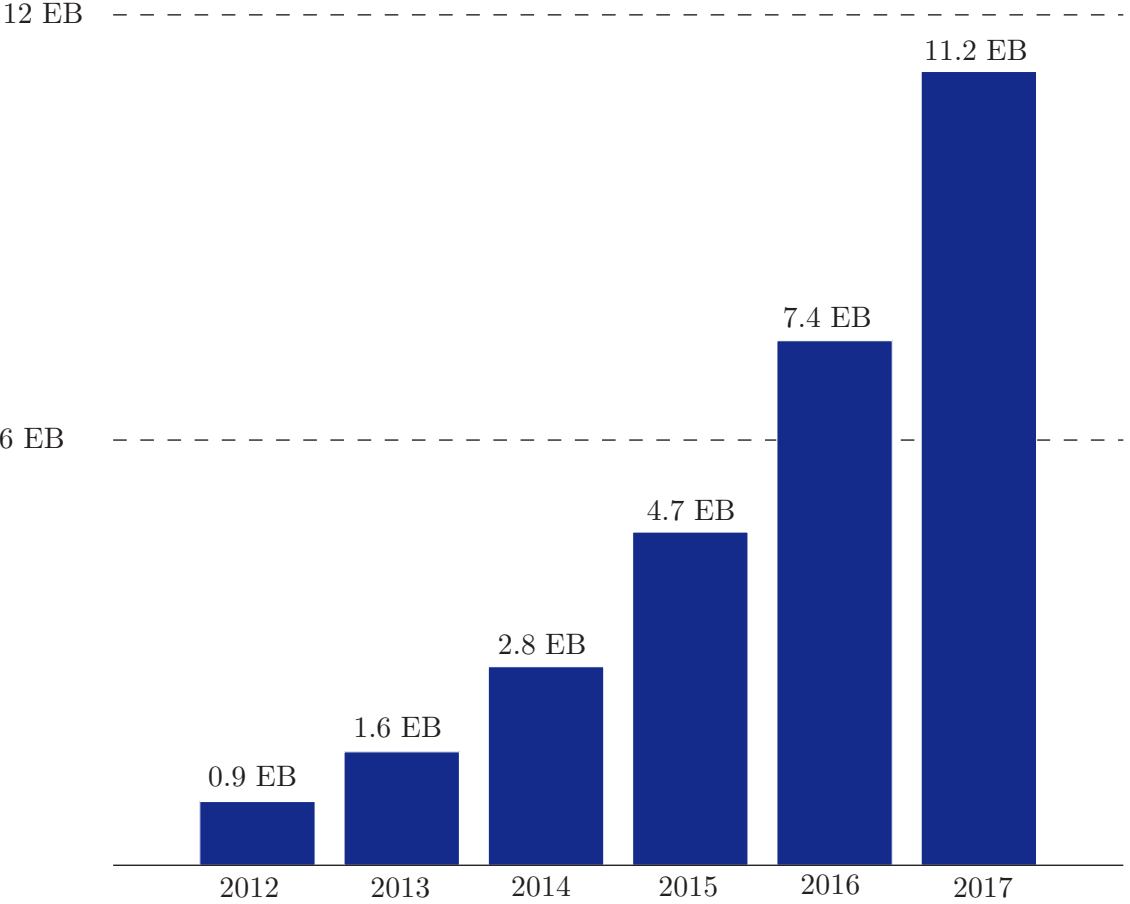

Figure 1.1: Average mobile data traffic per month during 2012 and 2013 and its predicted growth in the coming years. The figure is produced based on the data provided in [3, Figure 1].

\subsubsection{Adaptive Modulation and Coding}

Traditionally, the transmission parameters (modulation format and channel code) were kept fixed during data transmission. With adaptive modulation and coding (AMC), the transmitter selects the transmission parameters adaptively based on the instantaneous channel condition [4]. That is, when the communication link is good, the base station uses higher order modulation and a higher rate code and vice versa.

\subsubsection{Channel Dependent Scheduling}

Since in a wireless system, there are many users that request services from a base station and since the channels of the individual users change independently of each 
other, there is almost always a user whose channel is near its peak. By scheduling the user that has the best channel quality, a higher system throughput can be achieved. This is referred to as multi-user diversity in literature [5]. This technique along with AMC is actually a way to change the channel fading from a "foe" into a "friend". In other words, instead of considering channel fading to be something bad that needs to be overcome, it is regarded as a tool to achieve higher data rate.

\subsubsection{Hybrid Automatic Repeat Request}

In many applications, the receiver needs to receive the packets without error. A classical approach to support error-free transmission is the automatic repeat request $(A R Q)$ mechanism [6]. In an ARQ scheme, the receiver discards the erroneously received packets and requests retransmission. However, despite the fact that the received packet was not possible to be decoded, it still contains information which is lost by discarding the erroneously received packets. In Hybrid-ARQ (HARQ), instead of discarding the erroneously received packets, the receiver will store it in a buffer memory and later, combine it with the packets from the retransmission to obtain a single, combined packet which is more reliable than its constituents [7].

\subsubsection{Multiple Input Multiple Output (MIMO)}

By using more than one antenna at the transmitter or at the receiver, one can increase the strength of the received signal at the receiver. This is referred to as transmitter or receiver "diversity". By using two or more antennas both at the transmitter and at the receiver, there is also the possibility to use spatial multiplexing and consequently to enhance the system throughput [8]. This is typically referred to as multiple input multiple output (MIMO).

\subsection{Control Signaling}

No practical multiple access systems can be implemented without some sort of "control signaling" in the higher levels. The control signaling is referred to sending control information that is necessary to establish and/or maintain the connection. In the early wireless communication systems, the main task of the system was to provide a reliable voice connection between two entities as well as some simple services such as text messages. These services usually require low data rate and they impose low control signaling overhead on the system. For instance to establish a voice connection, terminal $\mathrm{A}$ who initiates the connection, sends the call request along with the identity of the terminal B, to whom it wants to call. Terminal B is 
then "called" or "paged" over the paging channel and provided that it is not busy and can accept the connection, a specific channel is dedicated for the corresponding call. Once the connection is established, no more control signaling is required.

As discussed earlier, by introducing new packet oriented services, such as web browsing, there was a need for much higher data rate connections. To support such services, many techniques such as those given in Section 1.1 were developed. While these techniques enhance the achievable data rate, they impose a substantial signaling overhead on the system. For instance, the price of using AMC and opportunistic scheduling is that the users must be informed about the transmission parameters as well as the locations where their payload is located prior to the actual data transmission since otherwise they will not be able to correctly decode the information. Therefore, many practical systems dedicate some part of the channel resources to control signaling.

\subsection{Thesis Overview}

In this thesis, we are not interested in how the wireless systems have evolved nor in how these new techniques can enhance the performance, but rather we are interested in studying how these new coming technologies have affected the system structure. More specifically, we are interested in studying how control signaling associated with the deployment of these new techniques is done. We consider different aspects of control signaling and propose some novel schemes for improving it. The dissertation is split into two parts. In the first part, the focus is on the transmission of scheduling assignments. In the second part, the focus is on improving the "blind decoding" process that is used to achieve adaptive coding and modulation in transmission of control information.

The thesis is a collection of seven included papers. The first part is comprised of four papers and the second part consists of three. Before we study the papers in more details, we give an introduction and an overview of the research related to control signaling. More precisely, the rest of this introductory part is organized as follows. We will first describe High Speed Packet Access (HSPA) and 3GPP Long Term Evolution (LTE) systems as two examples of how control signaling is implemented in practice in the next chapter. In Chapter 3, we will visit some of the research work related to the control signaling. Finally in Chapter 4, the specific contributions of the thesis will be listed, and in Chapter 5 the conclusions and some possible directions for the extension of the dissertation are discussed. 


\section{Chapter 2}

\section{Control Signaling in Wireless Multiple Access Systems}

We start this chapter with a very brief overview of the HSPA system, since HSPA is considered as the third generation $(3 \mathrm{G})$ wireless access technology. We then turn our attention to LTE, which is known also as the forth generation $(4 \mathrm{G})$ wireless access technology, and describe how the control signaling is implemented in LTE in more detail. The reason that we consider LTE in more detail is the fact that many of our results have been evaluated on LTE-like systems and hence a good understanding of LTE structure is important.

\subsection{High Speed Packet Access (HSPA)}

HSPA is considered to be the evolution of Wideband Code Division Multiple Access (WCDMA) and was introduced to boost the performance of WCDMA. A complete treatment of WCDMA is beyond the scope of this thesis. Interested readers are referred to [9-11]. Since WCDMA uses code-division multiple access (CDMA) [12] as the communication method, the channel resources, that can be assigned to the terminals, are basically code and power. Each user is assigned a part of a channelization code that is used as the spreading code at the call setup. During a packet-data call, the code assignment for a user does not change (unless the transmission is reconfigured). However, there is the possibility for power adaptation and power control commands are transmitted during the call. These control commands do not put an excessive signaling overhead on the system.

HSPA consists of two major components: (i) High-Speed Downlink Packet Access (HSDPA) [13] and (ii) High-Speed Uplink Packet Access (HSUPA) [14]. As their 
names suggest, HSDPA and HSUPA were developed to enhance the performance of WCDMA downlink and uplink, respectively. The key technology in both HSDPA and HSUPA is the introduction of a so-called shared-channel transmission. The shared-channel transmission denotes some part of the physical channel resources (power and part of code space), which is shared dynamically between users in time. In other words, a large part of the channel resources is assigned to a single user during a period of time, allowing for a higher data rate transmission. The scheduling assignments are valid for a duration of one transmission time interval (TTI) which is $2 \mathrm{~ms}$ in HSPA. In order to achieve higher data rates, channel dependent scheduling with AMC is implemented in HSPA. That is, the shared channel is assigned to a user where her channel condition is good, which consequently allows for opting for a higher AMC level. Therefore, in order to support successful transmission, the terminals need to receive the control information every $2 \mathrm{~ms}$. This is done via a high-speed shared control channel which carries the necessary information about spreading code, modulation format and coding rate to the terminals in the cell. As opposed to WCDMA, this imposes an extensive control signaling overhead on the system.

\section{$2.2 \quad$ A Brief Overview of LTE Downlink}

As discussed earlier in Section 2.1, HSPA was designed to improve the performance of WCDMA. Therefore, HSPA needs to be backward compatible to the existing WCDMA structure. In parallel to HSPA, 3rd Generation Partnership Project (3GPP) introduced a new multiple access system known as Long-Term Evolution (LTE). LTE targets higher performance goals compared to HSPA and it does not need to be backward compatible with the existing structure. However, LTE has to be more flexible in terms of bandwidth and it should be able to operate even in non-contiguous frequency bands [2].

We will first define the basic terminologies that are necessary for the upcoming discussions. Then we will study how the above techniques are implemented in LTE and how the control channel is designed to support successful data transmission. It is worth mentioning that the following description is very brief and does not cover every detail of the LTE implementation. For a detailed description, the readers are referred to [2] and the references therein.

LTE uses orthogonal-frequency division multiple access (OFDMA) [15] as the communication method in the downlink with the following specifications [16]. The subcarrier spacing is $15 \mathrm{kHz}$. The communication is done in frames of length $10 \mathrm{~ms}$. Each frame is divided into 10 equally sized subframes, hence the duration of a subframe is $1 \mathrm{~ms}$. Each subframe is further divided into two equally long slots of duration $0.5 \mathrm{~ms}$. LTE allows for two choices of cyclic prefix length: (i) normal cyclic prefix, and (ii) extended cyclic prefix. The main purpose of having an extended cyclic prefix is to enable a satisfactory operation when the channels have a 


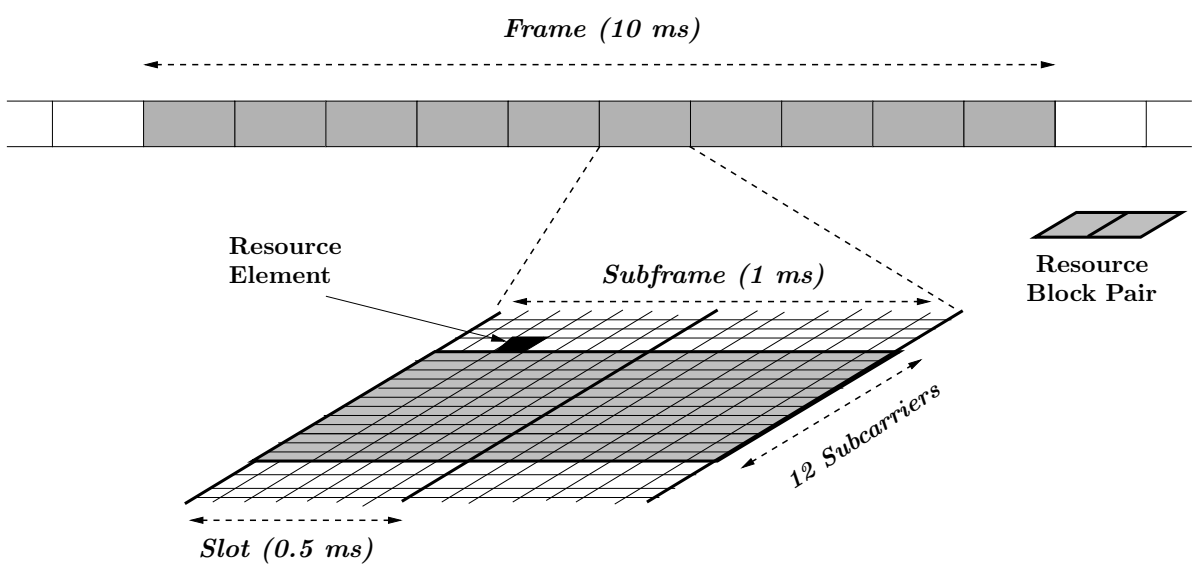

Figure 2.1: Physical layer channel resources in LTE.

huge delay spread. Each slot consists of 7 or 6 OFDM symbols, in the normal cyclic prefix mode and in the extended cyclic prefix mode, respectively. In the LTE context, one subcarrier during one OFDM symbol is called a resource element. Also, a collection of 12 consecutive subcarriers in each slot is called a resource block. As we shall see later, the minimum scheduling granularity in LTE consists of two resource blocks in each subframe which is referred to as a resource block pair [2, pp. 323-324]. Figure 2.1 illustrates the time/frequency domain structure of the LTE downlink.

\subsubsection{Channel Dependent Scheduling and Rate Adaptation}

In LTE, the scheduling is made at the beginning of each subframe. In contrast to HSPA, where the scheduler can exploit the channel variations only in time, LTE can exploit the variations both in time and in frequency, since the communication is based on OFDMA. However, to reduce the signaling overhead, the minimum scheduling granularity is one resource block pair. Payload data is transmitted in the form of transport blocks. AMC is performed by the scheduler, using different transport block formats. Each transport block format determines the AMC parameters that are used for the transmission.

\subsubsection{HARQ with Soft Combining}

To each transport block, a cyclic redundancy check (CRC) of length 24 bits is attached [17]. The attached CRC is used to determine whether the received transport block is in error or not. If no error is detected, the receiver transmits a positive 
acknowledgment (ACK) to the transmitter and passes the transport block to the upper layers. In the case of detected error(s), the receiver sends a negative acknowledgment (NACK) to the transmitter, but stores the transport block in its buffer for further processing. In order to facilitate a fast retransmission mechanism, the ACK/NACK messages should be transmitted to the transmitter as fast as possible. On the other hand, the terminals need to have enough time to perform a decoding attempt. In LTE, to support a fast HARQ mechanism and at the same time to give enough time to the terminals, the ACK/NACK messages are transmitted after $4 \mathrm{~ms}$. In other words, the ACK/NACK message corresponding to the transport block transmitted at subframe $n$, is sent in subframe $n+4$. The receiver uses soft combining [18] of the received packets to gain more reliable data in the case of retransmission.

\subsubsection{Support for Multiple Antennas}

In LTE, multiple antenna technology is supported at both the transmitter and the receiver [19]. Multiple antennas at the receiver facilitate transmit diversity, whereas multiple antennas at the transmitter support beam-forming as well. In the case of multiple antennas at the transmitter and at the receiver, that is in case MIMO is used, there is a possibility to transmit multiple data streams and hence this is a key technique to improve the system spectral efficiency in LTE.

\subsection{The Control Channel in LTE Downlink}

As we have seen, the scheduling decisions are made at the beginning of each subframe. This means that the scheduling decision as well as the information about AMC parameters used for the data transmission need to be sent to all scheduled users every $1 \mathrm{~ms}$. In addition, the HARQ ACK/NACK messages corresponding to the uplink transmission also have to be sent in each subframe. To support this, up to the first three OFDM symbols in each subframe can be used for control signaling. The size of the control region may be changed from subframe to subframe. The reason behind this is to adjust to the traffic situation. More precisely, when there are many users scheduled for the transmission in the subframe, that is in high traffic situations, then the size of the control region is 3 OFDM symbols, allowing to accommodate the control information of all users. In contrast, when there are few users scheduled for payload reception in the subframe, then the control region can be reduced to 1 OFDM symbol, allowing for a better usage of channel resources. The reason for having the control region at the beginning of each subframe is as follows: the scheduled users can find the information about the resource allocation and the transmission parameters as early as possible. Therefore, a terminal does not need to wait until the entire subframe transmission is over to find the transmission 


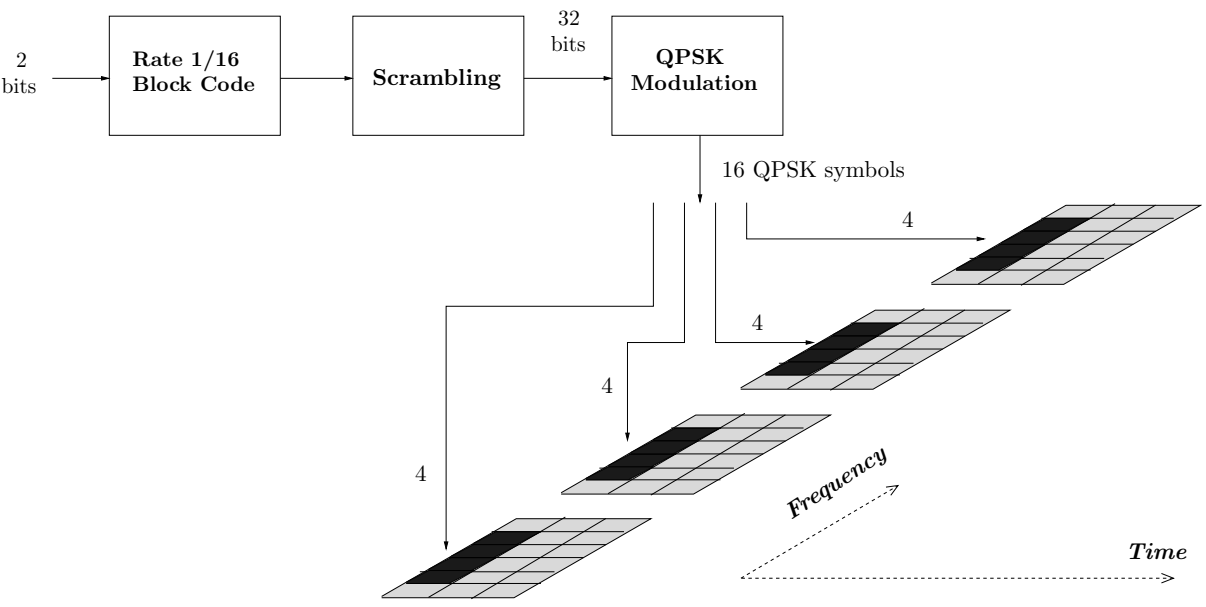

Figure 2.2: The PCFICH Structure. This figure is freely reproduced from [2, pp. 333].

parameters and hence it can start the decoding process right after the transmission of the control information. This helps the terminals to reduce the decoding delay. The control region in LTE consists of three different physical channel types, that we describe briefly in the following subsections.

\subsubsection{Physical Control Format Indicator Channel}

The physical control format indicator channel (PCFICH) is used to signal the size of the control region. Since up to three OFDM symbols may be used for the control region, two bits are required for representing the size of the control region. Therefore, two information bits are transmitted on the PCFICH. Correct decoding of the PCFICH is essential, since it determines the size of the control region and consequently the start of the data region. If a terminal fails to decode the PCFICH, it neither knows where to look for the control information nor where the data region starts.

Figure 2.2 illustrates how the PCFICH is transmitted in the control region. The two information bits are first encoded by a block code of rate 1/16 to gain enough error protection. The coded bits are then scrambled with a cell and subframe specific scrambling code. This is used to randomize the inter-cell interference. Then the scrambled bits are modulated using quadrature phase shift keying (QPSK) modulation [20] and the resulting QPSK symbols are mapped into 16 resource elements. Since the size of the control information is not known until the successful decoding of the PCFICH, the modulated symbols are mapped into the first OFDM 
symbol. More specifically, they are mapped into blocks of so-called resource element groups each consisting of four consecutive resource elements. To achieve frequency diversity, the corresponding resource element groups are separated well enough in frequency. It is also worth mentioning that the location of resource element groups depends on the cell identity. This is helpful to reduce the interference between neighboring cells.

\subsubsection{Physical HARQ Indicator Channel}

The physical HARQ indicator channel (PHICH) is used to transmit the HARQ acknowledgments of the uplink transmission. Each PHICH carries the acknowledgment message (one information bit) of one uplink data session. The mapping of PHICH:s onto resource elements is subject to a certain structure which, as in the PCFICH mapping, is based on resource element groups. More precisely, several $\mathrm{PHICH}: \mathrm{s}$ are first assigned to a certain $\mathrm{PHICH}$ group ${ }^{1}$. The PHICH:s in each group are code multiplexed onto 3 resource element groups as illustrated in Figure 2.3. Each $\mathrm{PHICH}$ is first encoded using rate $1 / 3$ repetition encoding. The coded bits are then modulated using binary phase shift keying (BPSK) using either in-phase (I) or quadrature (Q) branches [20]. The resulting symbols are then spread via an orthogonal code of length 4 . The resulting 12 symbols of all such branches (corresponding to one $\mathrm{PHICH}$ ) are then added together to form 12 QPSK modulated symbols which are then mapped onto 3 resource element groups. Note that once again, the cell specific scrambling is done to randomize the interference and that the resource element groups are located far apart to achieve frequency diversity.

\subsubsection{Physical Downlink Control Channel}

The physical downlink control channel (PDCCH) is used to transmit the downlink control information (DCI). DCI includes many control information types. Most importantly, downlink scheduling assignments, information about what transport block format is used (which, as discussed earlier, determines the AMC parameters that are used for payload transmission), control information regarding spatial multiplexing (if MIMO is used) and HARQ information are included in DCI. It is worth mentioning that there exist different DCI formats each having different size [19]. The reason behind that is to give the opportunity to trade the scheduling granularity and the flexibility in choosing transport block formats for signaling overhead. For instance, one of the DCI formats, namely DCI format 1C, allows only QPSK modulation, has no HARQ support and hence it has a smaller size compared to the other DCI formats. Therefore, this format is useful when the control channel is congested due to its smaller size.

\footnotetext{
${ }^{1}$ In the case of normal cyclic prefix operation mode, 8 PHICH:s forms one PHICH group [2].
} 


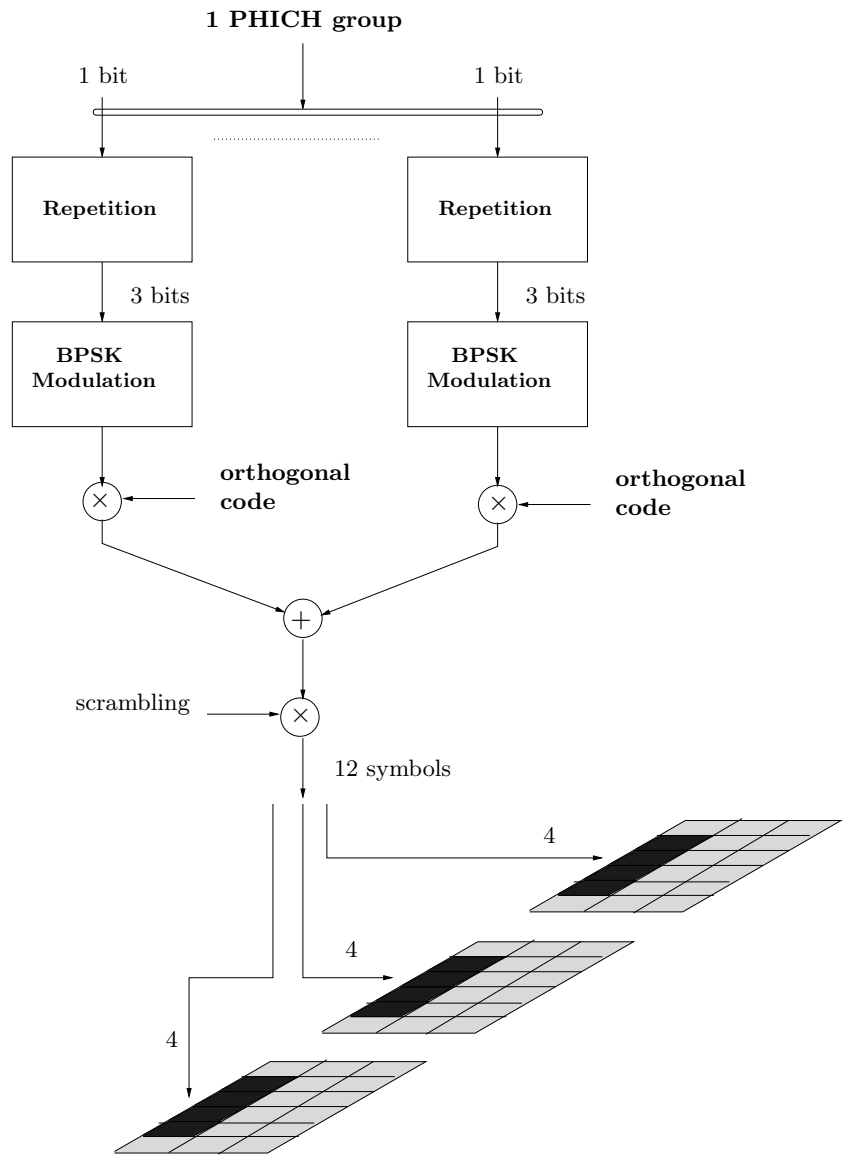

Figure 2.3: The PHICH Structure. This figure is freely reproduced from [2, pp. 337].

Since there might be several users scheduled for payload transmission in the subframe, and since each PDCCH carries one message according to one of the available DCI formats, there might be several simultaneous PDCCH transmissions within each subframe. Each PDCCH transmission is intended to one of the scheduled users.

Figure 2.4 illustrates the PDCCH processing in LTE. A CRC of length 16 is attached to the control information intended to each user [17]. The attached CRC is used not only to determine the correct reception of control information but also to pinpoint the user to whom the DCI is intended. This is done by using a user-specific CRC. In other words, instead of explicitly signaling the identity of the user, the identity of the user is embedded in the CRC. As we will see shortly, the attached CRC is also used as a means to achieve adaptive coding and modulation. After the CRC 


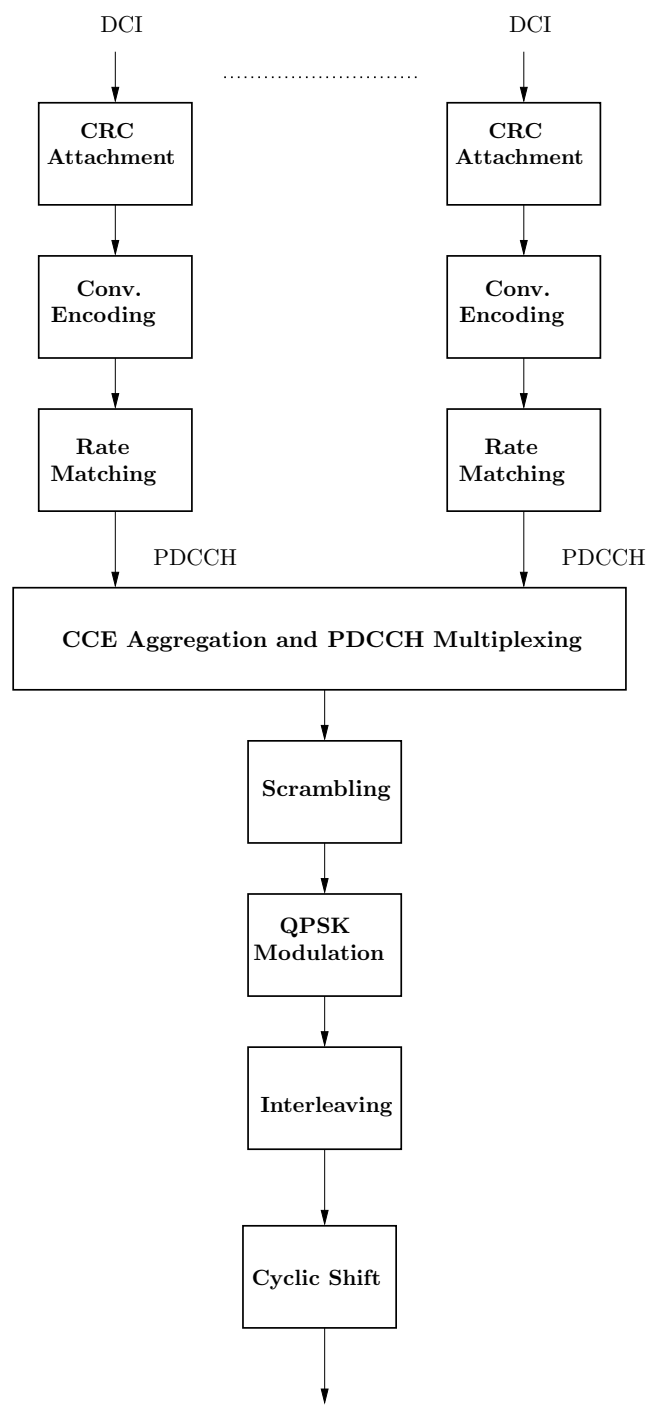

Figure 2.4: The PDCCH Structure. This figure is freely reproduced from [2, pp. 353].

attachment, the output bits are encoded using a tail-biting rate $1 / 3$ convolutional code $[21]$.

The mapping of coded bits onto the resource elements is subject to a certain structure. More precisely, every PDCCH is mapped to 1, 2, 4 or 8 control channel elements (CCE:s). Each CCE consists of 9 resource element groups (that is 36 
resource elements). The number of CCE:s used for PDCCH transmission is determined not only from the DCI message size, the number of OFDM symbols dedicated to the control region and the cell bandwidth, but also from the instantaneous channel conditions. This is used to achieve AMC on the control channel. More precisely, the coded bits obtained from the convolutional code are matched (through puncturing in the case of good channel conditions and through repetition in the case of poor channel conditions) to fit the number of CCE:s reserved for the transmission of the corresponding PDCCH. After allocation of the PDCCH:s to the CCE:s, the bits are scrambled using a cell specific and subframe specific sequence number as before, to reduce the inter-cell interference. The resulting bits are QPSK modulated and mapped to the corresponding resource elements.

\subsubsection{Blind Decoding of PDCCH:s}

As we have seen, the number and/or the location of CCE:s used for the PDCCH transmissions ${ }^{2}$ are not known to the users in advance. In order to find its control information, a terminal tries to blindly decode the incoming control information assuming different combinations of CCE:s and check for the CRC. If the CRC checks after a decoding attempt, then the terminal assumes that the corresponding PDCCH was intended for her and that the control information was decoded correctly. If the CRC does not match, then the terminal tries a new combination/location of CCE:s. In order to keep the number of decoding attempts low, LTE uses a so-called search space for each terminal. The search space determines the location/combination of CCE:s that the terminal needs to monitor for a possible control information. The size of the search space is 44 in LTE [2, pp. 358].

\footnotetext{
${ }^{2}$ This is referred to as CCE aggregation in the LTE context.
} 


\section{Chapter 3}

\section{Literature Overview on Control Signaling}

Although control signaling has significant impact on the overall system performance, it had drawn little attention in the literature until recently. The main motivation of the research work in control signaling is to improve the efficiency by, for instance, reducing the amount of control information that needs to be communicated, or by decreasing the number of resources required for the transmission of this control information. There are various ways for categorizing the research on control signaling. One such categorization is based on whether the research is applicable in the downlink or in the uplink. Another categorization is based on the specific part of the control signaling that the research focuses on (such as how to send the scheduling assignments, or how to reduce the overhead associated with the transmission of HARQ acknowledgments, etc.) The research work can also be categorized based on the specific system that benefits from the research (for example if it is LTE specific, HSPA specific or IEEE 802.16e specific, etc.). In this chapter, we first give a general overview of the literature on control signaling. We then specify how the contributions of this dissertation are placed in correspondence to the other research in this area.

\subsection{Research Related to Signaling Overhead}

As discussed earlier, the techniques for boosting the system performance impose signaling overhead that needs to be taken into account when designing a wireless system. For example, to achieve multiuser diversity the scheduler needs to know the communication links of all the users. This information is typically obtained from 
the channel state information that is reported by each terminal on a feedback link. Achieving the perfect channel knowledge requires a significant amount of signaling in the feedback link. There is a body of literature studying the associated signaling overhead. For instance in $[22,23]$, the achievable sum rate with partial channel state information and with reduced signaling in the feedback link for OFDMA were studied. In [24-26], the authors proposed compression schemes for the channel state information that is transmitted in the feedback link. The authors in [24] also provided a comprehensive review of the research related to decreasing the amount of required feedback for acquiring channel state at the base station. Other examples in this area are [27-29], where new schemes are proposed that can achieve multiuser diversity but require limited amount of signaling overhead for reporting the channel state information in the feedback link.

In [30], a dynamic resource allocation technique for OFDMA systems was proposed that minimizes the quality of service (QoS) violation ratio. This ratio is defined as the fraction between the number of users whose quality of services are not satisfied to the total number of users. The proposed scheme therein requires little signaling overhead for the coordination. The idea is to split the resource allocation problem into two levels. In the first level, the base stations are divided into different inter-cell coordinator (ICC) groups. In the next level, the resources are allocated to the users in each cell. As opposed to the conventional scheme in which a central unit gathers all channel state information from all users to make the scheduling decisions, in the proposed scheme the scheduling decisions are made in a decentralized manner. The only information that is exchanged among the ICC groups is the total number of subcarriers required for supporting the data rate requirements of the users, and hence the proposed scheme requires limited signaling overhead for the coordination.

Another important aspect of signaling overhead associated with exploiting multiuser diversity is that the terminals need to be informed about the scheduling assignments prior to the actual payload transmission. This signaling overhead consumes significant part of channel resources in many situations [31,32]. Examples of papers addressing this problem are [33-35]. In [33], the effect of control signaling and outdated channel state information on the system performance was studied. The authors showed that the performance of dynamic resource allocation is reduced when there is a control signaling cost and also when the instantaneous channel knowledge is not present at the scheduler. In [34], two schemes for reducing the amount of signaling overhead, that concerns the transmission of scheduling assignments, are presented. The idea is to use the correlation that exists between the scheduling assignments in the successive subcarriers. That is, if a user has a good channel on a certain subcarrier, then it is very likely that she has good channels on the neighboring subcarriers as well, which can be used to compress the scheduling assignments more effectively. In [35], the author proposed semi-fixed scheduling assignments for voice over Internet protocol (VoIP) services that reduces the signaling overhead. The idea therein is to use the fact that voice services typically have predictable behavior which can be used for reducing the amount of control signaling. 
The price for exploiting adaptive modulation and coding (AMC), as in the case of exploiting multiuser diversity, is that the transmission parameters need to be sent to the users before the transmission. There is, however, many papers addressing this issue. The main idea is that the receiver tries blindly to identify the transmission parameters. For instance in $[36,37]$, different techniques for blind classification of the modulation format were studied, and in [38-40], different schemes for blindly identifying the channel code were considered. When it is known that the channel code has been chosen from a set of predefined candidate set, the receiver can use this a priori information for achieving a better blind channel code identification. This problem was addressed in [41-43].

Examples of the work related to specific wireless systems are [44-50]. In [44-46], the signaling overhead in IEEE 802.16e OFDMA systems was studied. For instance in [44], the authors proposed an algorithm for reducing the amount of control signaling for IEEE 802.16e OFDMA systems. The idea is to concatenate the payload data of each user with her control information in the coming frame. Since typically AMC is used only for the transmission of payload data (not control information), the proposed scheme can reduce the amount of signaling overhead. In [45], the authors proposed to use mapping with appropriate truncation and sort algorithm when scheduling the users and evaluated its performance using system simulations. In [46], the effect of persistent scheduling (in a round-robin fashion) on the system performance for IEEE 802.16e OFDMA systems were studied.

In [47-50], different aspects of control signaling in LTE systems were considered. More precisely in $[47,48]$, two methods for improving the error protection of physical uplink control channel (PUCCH) in LTE were proposed. These methods are based on complex-field coding [51] and on repetition across the two frequency bands used for the transmission of PUCCH, respectively. In [49], an efficient low complexity receiver for improving the performance of detection for PUCCH was proposed and in [50], a robust multiuser channel estimator and detector for PUCCH was described.

\subsection{Contributions of the Dissertation}

The dissertation is organized into two parts where in the first part the focus is on the transmission of scheduling assignments, and in the second part the focus is on improving the "blind decoding" process that is used to achieve adaptive coding and modulation in the transmission of control information.

More specifically, in the first part of the dissertation that comprises four included papers (Papers A-D), we first compare the two conventional schemes for control signaling using extensive system simulations. In doing so, we use practical assumptions on the scheduling algorithm as well as on the compression and transmission of the scheduling information. We then provide two schemes for reducing the amount 


\section{Research on Control Signaling}

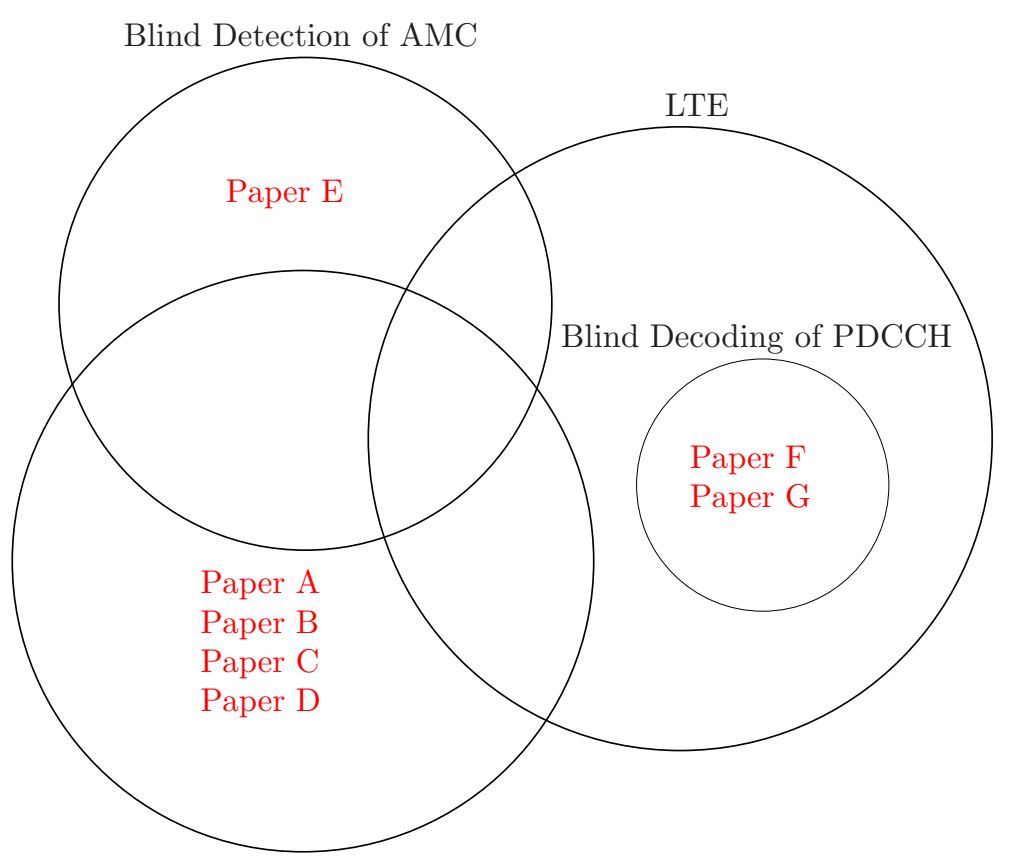

Transmission of Scheduling Assignments

Figure 3.1: The placement of the thesis contributions according to the research work related to control signaling.

of control signaling that concerns the transmission of scheduling assignments. The first scheme, which is reminiscent of source coding with side information, uses the knowledge that each user has about its own channel condition to compress the scheduling information more effectively. The second scheme uses the fact that in wireless multiple access systems, a user with a given channel condition can in principle decode the data intended to the users that have weaker channels. Therefore, the idea is to send the scheduling information of different terminals in a differential manner starting from the user with the weakest channel and letting all the terminals overhear the transmission of one another. Finally, in the last section of this part we use some of the recent results in information theory to form a general framework for the comparison of different control signaling schemes. We formulate an optimization problem that for a given desired error probability finds the minimum required number of channel uses for a given signaling scheme.

In the second part of the thesis, that contains three included papers (Papers E-G), we propose three schemes for reducing the complexity of the blind decoding process. 
The first one is a novel scheme for fast blind identification of channel codes. More precisely, we propose an efficient algorithm that for a given sequence of received symbols and a given linear channel code, finds the posterior probability that all the parity check relations of the code are satisfied. We then use this quantity to perform a sequential statistical hypotheses test that reduces the computational complexity of blind decoding. The idea in the second scheme is to broadcast a control message prior to the transmission of control information to instruct only a subset of the terminals (ideally only those terminals that have been scheduled for reception of payload data and hence benefit from performing a blind search attempt) to perform blind search decoding, which can be used for instance in LTE to reduce the complexity of the blind decoding process. Finally, in the third scheme we propose to split the CRC, used by the terminals to find their control information, into two parts and inject one part early in the control data stream so that the terminals can detect early if the current decoding attempt will be successful, which ultimately reduces the blind decoding complexity. The papers included in the dissertation are illustrated in Figure 3.1 in combination with the related work. In the next Chapter, the specific contributions and a summary of the papers will be given. 


\section{Chapter 4}

\section{Summary of Specific Contributions of the Dissertation}

As discussed earlier, the dissertation is comprised of seven included papers all in various ways related to the control signaling. In Paper A, we compare the two conventional schemes for control signaling using extensive system simulations, using practical assumptions on the scheduling algorithm as well as on the compression and transmission of the scheduling information. In Papers $\mathrm{B}$ and $\mathrm{C}$, we provide two novel schemes for reducing the amount of control signaling that concerns the transmission of scheduling assignments. In Paper D, we use recent results in information theory to form a general framework for the comparison of different control signaling schemes. In Papers E-G, we propose three schemes for reducing the complexity of the blind decoding process. The first one, presented in Paper E, is a novel scheme for fast blind identification of channel codes. The idea behind the second algorithm, presented in Paper $\mathrm{F}$, is to broadcast a control message prior to the transmission of control information to instruct only a subset of the terminals to perform blind search decoding, which reduces the complexity of the blind decoding process. Finally, in Paper G, we propose the third scheme for reducing the complexity of blind decoding by splitting the CRC, used by the terminals to find their control information, into two parts and injecting one part early in the control data stream so that the terminals can detect early if the current decoding attempt will be successful.

\subsection{Included Papers}

Brief summaries of the papers included in the thesis are given below. 


\section{Paper A: Comparison of Strategies for Signaling of Scheduling Assign- ments in Wireless OFDMA}

Authored by R. Moosavi, J. Eriksson, E. G. Larsson, N. Wiberg, P. Frenger and F. Gunnarsson.

Published in the IEEE Transactions on Vehicular Technology, Nov. 2010.

This paper considers transmission of scheduling information in OFDMA-based cellular communication systems such as 3GPP long-term evolution (LTE). These systems provide efficient usage of radio resources by allowing users to be scheduled dynamically in both frequency and time. This requires considerable amounts of scheduling information to be sent to the users. The paper compares two basic transmission strategies: transmitting a separate scheduling message to each user versus broadcasting a joint scheduling message to all users. Different scheduling granularities are considered, as well as different scheduling algorithms. The schemes are evaluated in the context of the LTE downlink using multiuser system simulations, assuming a full-buffer situation. The results show that separate transmission of the scheduling information requires a slightly lower overhead than joint broadcasting, when proportional fair scheduling is employed and the users are spread out over the cell area. The results also indicate that the scheduling granularity standardized for LTE provides a good trade-off between scheduling granularity and overhead.

\section{Paper B: Reducing Physical Layer Control Signaling Using Mobile- Assisted Scheduling}

Authored by R. Moosavi and E. G. Larsson.

Published in the IEEE Transactions on Wireless Communications, Jan. 2013.

We present a scheme for reducing the part of the downlink signaling traffic in wireless multiple access systems that contains scheduling information. The theoretical basis of the scheme is that the scheduling decisions made by the base station are correlated with the CSI reports from the mobiles. This correlation can be exploited by the source coding scheme that is used to compress the scheduling maps before they are sent to the mobiles. In the proposed scheme, this idea is implemented by letting the mobiles make tentative scheduling decisions themselves, and then letting the base station transmit "agreement maps" instead of raw scheduling maps to the mobiles. The agreement maps have lower entropy and they require less resources to be transmitted than the original scheduling maps do. The improvement can be substantial. We also model the task of finding the optimal scheduling assignments according to the proposed scheme as a combinatorial optimization problem and present an efficient algorithm to find the optimal solution. 


\section{Paper C: Differential Signaling of Scheduling Information in Wireless Multiple Access Systems}

Authored by R. Moosavi, J. Eriksson and E. G. Larsson.

Published at the IEEE Global Communications Conference (GLOBECOM), Dec. 2010 .

This paper considers the control signaling on the downlink in wireless multiple access systems, with focus on the part of the control signaling that carries information on the user's time/frequency scheduling assignments. A new idea is presented to reduce the amount of channel resources needed for this signaling. The idea is to exploit the fact that provided that only one single user is scheduled on each channel resource, then the different users' scheduling assignments are correlated. This correlation can be exploited by encoding the scheduling information differentially. In order to recover the scheduling information, a user must then decode the scheduling information of some of the others. This is possible, because on the downlink, all users can hear the transmission by the base station so that users with a high SNR may decode the control signaling sent to users with a lower SNR. We present a practical scheme to exploit this idea. Both analytical analysis and numerical examples illustrate that the proposed technique can provide a substantial reduction in signaling traffic.

\section{Paper D: Optimized Encoding of Scheduling Assignments Using Finite Blocklength Coding Bounds}

Authored by R. Moosavi and E. G. Larsson.

Submitted to the IEEE Wireless Communications Letters.

We provide an analytical framework for optimizing the resources required for signaling of control information in wireless multiple access systems. In doing so, we use recent results in information theory, namely a new bound on the achievable rate in the finite blocklength regime by Polyanskiy. We formulate optimization problems for finding the minimum required number of channel uses such that the overall error probability in decoding the control information is below a given threshold for three different control signaling schemes.

\section{Paper E: Fast Blind Recognition of Channel Codes}

Authored by R. Moosavi and E. G. Larsson.

Submitted to the IEEE Transactions on Communications.

We present a fast algorithm that, for a given input sequence and a linear channel code, computes the syndrome posterior probability (SPP) of the code, i.e., the 
probability that all parity check relations of the code are satisfied. According to this algorithm, the SPP can be computed blindly, i.e., given the soft information on a received sequence we can compute the SPP for the code without first decoding the bits. We show that the proposed scheme is efficient by investigating its computational complexity.

We then consider two scenarios where our proposed SPP algorithm can be used. The first scenario is when we are interested in finding out whether a certain code was used to encode a data stream. We formulate a statistical hypothesis test and we investigate its performance. We also compare the performance of our scheme with that of an existing scheme. The second scenario deals with how we can use the algorithm for reducing the computational complexity of blind decoding process, the process that, for instance, is used by terminals in LTE for detection of control information. We propose a heuristic sequential statistical hypotheses test to use the fact that in real applications, the data arrives sequentially, and we investigate its performance using system simulations.

\section{Paper F: Fast Identification of Control Signaling Aided by Please- Decode-Blindly (PDB) Messages}

Authored by R. Moosavi and E. G. Larsson.

Published at the IEEE Swedish Communication Technologies Workshop (SweCTW), Oct. 2012.

Blind decoding of control information is used in some wireless multiple access systems such as LTE to achieve adaptive modulation and coding, as well as to address the multiple access problem on the control channel. Blind decoding incurs high computational complexity in mobile terminals. In this paper, we describe a scheme to reduce the computational complexity associated with the blind decoding. The main idea is to broadcast a "please-decode-blindly" message to all terminals that are eligible for scheduling, to instruct a subset of the terminals to perform the blind search. We propose two schemes to implement our idea and we investigate their performances via system simulations.

\section{Paper G: Complexity Reduction of Blind Decoding Schemes Using CRC Splitting}

Authored by J. Eriksson, R. Moosavi and E. G. Larsson.

Published at the IEEE Global Communications Conference (GLOBECOM), Dec. 2012.

Blind decoding, used on control channels of some multi-user wireless access systems, is a technique for achieving adaptive modulation and coding. The idea is to adapt the modulation and coding scheme to the channel quality but instead of signaling the 
parameters used explicitly, the receiver blindly tries a number of fixed parameter combinations until a successful decoding attempt is detected, with the help of a cyclic redundancy check. In this paper we suggest a new method for reducing the complexity and energy consumption associated with such blind decoding schemes. Our idea is to use a mini-CRC injected early in the data stream to determine if the current decoding attempt is using the correct modulation and coding parameters. We analyze and exemplify the complexity gain of this approach and also investigate the impact of the rearrangement of the CRC scheme in terms of the probability of undetected error. The presented results for the complexity gain are promising and the impact on the error detection capability turns out to be small if any.

\subsection{Not Included Papers}

The following papers contain work done by the author but are not included in the thesis, because either they do not fit within the main scope of the dissertation, or they were the earlier versions of the journal publications included in the dissertation.

- J. Eriksson, R. Moosavi, E. G. Larsson, N. Wiberg, P. Frenger and F. Gunnarsson, "On coding of scheduling information in OFDM," in Proc. of IEEE VTC, pp. 1-5, Apr. 2009.

- R. Moosavi and E. G. Larsson, "Reducing downlink signaling traffic in wireless systems using mobile-assisted scheduling," in Proc. of IEEE GLOBECOM, pp. 1-5, Dec. 2010.

- R. Moosavi and E. G. Larsson, "A fast scheme for blind identification of channel codes," in Proc. of IEEE GLOBECOM 2011, Dec. 2011.

- E. G. Larsson and R. Moosavi, "Piggybacking an additional lonely bit on linearly coded payload data," IEEE Wireless Commun. Letters, vol. 1, pp. 292-295, Aug. 2012. 


\section{Chapter 5}

\section{Conclusions and Future Research Directions}

As discussed in the previous chapters, many techniques designed for improving the system performance impose signaling overhead on the system. Examples of these techniques are multiuser diversity (by assigning the resources to the users based on their instantaneous channel conditions) and adaptive modulation and coding (AMC), where the price of exploiting them is the corresponding required signaling overhead. This is so because, in order to be able to perform opportunistic scheduling and AMC, the scheduler needs a non-causal knowledge of the channel conditions of all users. This information is often hard to obtain and requires significant amount of control signaling in a reverse link where each user reports his/her channel state information back to the scheduler. Additionally once the scheduling is done, the pertinent information on resource allocations and AMC parameters need to be sent to each terminal, since otherwise no communication is feasible. This also requires additional control signaling.

As the examples above illustrate, control signaling is an important part of any wireless multiple access system. However, this problem seems to be often overlooked in the literature. In this dissertation, we have studied different aspects of it and proposed some techniques for improving it. We next provide some conclusions that can be drawn from this dissertation, followed by some possible directions for the future research.

\subsection{Conclusions}

The key points associated with the first part of the dissertation, where we focus on the part of the signaling overhead that concerns the transmission of scheduling 
assignments, are:

- The system performance is highly dependent of the choice of scheduling algorithm as well as the method used for encoding and transmission of control information. As a rule-of-thumb, when the users have very different channel conditions, then it is better to send the scheduling information separately. In contrast, when there are no channel variations between different users, then it is better to compress the scheduling information jointly and broadcast the corresponding compressed information to all users.

- Scheduling with the finest granularity, despite the fact that it provides the opportunity to exploit the most multiuser diversity both in time and in frequency, results in the worst performance for both the system-throughput maximizing scheduler and for the proportional fair scheduler. The signaling overhead due to the transmission of the scheduling assignments consumes a significant amount of channel resources.

- When encoding the scheduling information, we should use all the information that the users have regarding the scheduling decisions for more effective compression of the scheduling assignments. One such information, is the correlation that exists between the scheduling decisions and the channel state information reported by the terminals. We have suggested a scheme that can reduce the control signaling overhead by about $20 \%$.

- In the wireless multiple access systems, since users with good channel conditions can decode the data intended to those with weaker channels, we can transmit the scheduling assignments in a differential manner which requires less channel resources. We have proposed an algorithm that exploits this and can achieve the compression that is obtained when the scheduling assignments are jointly encoded and broadcast.

In the second part of the dissertation, we have studied the problem of achieving AMC without additional signaling overhead. We have presented a fast algorithm for blindly recognizing which channel code from a candidate set that was used to encode a data stream. The proposed algorithm uses the fact than any linear code satisfies a certain set of parity check relations. Our algorithm obtains the probabilities that all parity check constraints are satisfied, called the syndrome posterior probability (SPP) of the code, for all code candidates and then compares these probabilities. We also proposed a sequential hypothesis test that makes decisions before collecting all available data, hence saving computational complexity. Under typical operating conditions, the algorithm identifies the correct code (out of 16 candidates) in $99 \%$ of the cases by observing less than 50 samples, at an SNR of 4 dB. We have also proposed two schemes that can be used in LTE for facilitating a better blind decoding of PDCCH. Numerical results showed that the two proposed scheme can save significant computational complexity. 


\subsection{Future Research Directions}

This dissertation may be extended in several ways. Below is a list of possible directions:

- Most of the simulation results presented in this thesis are with the assumption of single antenna transmission. While in many cases, it is straightforward to extend the proposed algorithms to multiple antenna systems, a comprehensive study of that is a possible extension of this work.

- Another interesting direction is to study the system performance when the proposed schemes (specially those proposed in Papers B and C) are used simultaneously. This can be done under the assumptions used in Paper D, i.e., using entropy for compression along with the finite blocklength coding bounds for the transmission.

- As will be mentioned later, using the proposed scheme in Paper E, it is possible to facilitate entirely blind multiple access, which is based on the terminals blindly recognizing their payload data. In this case, the base station would not signal any explicit control information or AMC parameters at all. A full study of this is another direction for future research.

- Finding the optimal grouping for the proposed scheme in Paper F is yet another future research direction. As another extension of this work, one may consider the scheduling problem that achieves a certain quality of service (QoS) while at the same time also takes the number of blind attempts into account.

- As systems exploiting hundreds of antennas seem to be the main driver for the next generation wireless multiple access systems and in combinations with the first item mentioned earlier, it is very interesting to study the design of control channel for systems with many antennas. 


\section{References}

[1] G. Weightman, Signor Marconi's Magic Box: The Most Remarkable Invention of the 19th Century \& The Amateur Inventor Whose Genius Sparked a Revolution, Da Capo Press, 2003.

[2] E. Dahlman, S. Parkvall, J. Sköld and P. Beming, 3G Evolution HSPA and LTE for Mobile Broadband, 2nd Edition Academic Press, 2008.

[3] Cisco Visual Networking Index: Global Mobile Data Traffic Forecast Update, 2012-2017, Feb. 2013.

[4] A. J. Goldsmith and S. G. Chua, "Adaptive coded modulation for fading channels," IEEE Trans. Commun., vol. 46, no. 5, pp. 595-602, May 1998.

[5] P. Viswanath, D. N. C. Tse and R. Laroia, "Opportunistic beamforming using dumb antennas," IEEE Trans. Inf. Theory, vol. 48, pp. 1277-1294, Jun. 2002.

[6] S. Lin, D. J. Costello and M. Miller, "Automatic-repeat-request error-control schemes," IEEE Commun. Mag., vol. 22, pp. 5-16, Dec. 1984.

[7] J. F. Cheng, "Coding performance of hybrid ARQ schemes," IEEE Trans. Commun., vol. 54, pp. 1017-1029, Jun. 2006.

[8] D. Tse and P. Viswanath, Fundamentals of Wireless Communication, Cambridge University Press, 2005.

[9] H. Holma and A. Toskala, WCDMA for UMTS: Radio Access for Third Generation Mobile Communications, John Wiley \& Sons, Chichester, UK, 2000.

[10] E. Dahlman, B. Gudmundsson, M. Nilsson and J. Sköld, "UMTS/IMT-2000 based on wideband CDMA," IEEE Commun. Mag., pp. 70-80, Sep. 1998.

[11] E. Dahlman, P. Beming, J. Knutsson, F. Ovesjö, M. Persson and C. Roobol, "WCDMA - the radio interface for future mobile multimedia communications," IEEE Trans. Veh. Technol., vol. 47, pp. 1105-1118, Nov. 1998.

[12] T. S. Rappaport, Wireless Communications Principles and Practice, PrenticeHall, 2002. 
[13] 3GPP TS 25.308, “3rd Generation Partnership Project; Technical Specification Group Radio Access Network; High Speed Downlink Packet Access (HSDPA); Stage 2 (Release 7)," Jun. 2009.

[14] 3GPP TS 25.309, "3rd Generation Partnership Project; Technical Specification Group Radio Access Network; FDD Enhanced Uplink; Overall description; Stage 2 (Release 6)," Mar. 2006.

[15] R. V. Nee and R. Prasad, OFDM for Wireless Multimedia Communications, Artech House Publisher, UK, 2000.

[16] 3GPP TS 36.211, "3rd Generation Partnership Project; Technical Specification Group Radio Access Network; Evolved Universal Terrestrial Radio Access (E-UTRA); Physical Channels and Modulation (Release 9)," Mar. 2010.

[17] 3GPP TS 36.212, "3rd Generation Partnership Project; Technical Specification Group Radio Access Network; Evolved Universal Terrestrial Radio Access (E-UTRA); Multiplexing and channel coding (Release 9)," Sep. 2010.

[18] P. Frenger, S. Parkvall and E. Dahlman, "Performance comparison of HARQ with Chase combining and incremental redundancy for HSDPA," in Proc. of IEEE VTC, pp. 1829-1833, Oct. 2001.

[19] 3GPP TS 36.213, "3rd Generation Partnership Project; Technical Specification Group Radio Access Network; Evolved Universal Terrestrial Radio Access (E-UTRA); Physical layer procedures (Release 9)," Jun. 2009.

[20] J. G Proakis and M. Salehi, Digital Communication, 5th Edition McGraw-Hill International Edition, 2008.

[21] R. Johannesson and K. Sh. Zigangirov, Fundamentals of Convolutional Coding, IEEE Press, 1999.

[22] S. H. Hur and B. D. Rao, "Sum rate analysis of a reduced feedback OFDMA downlink system employing joint scheduling and diversity,", IEEE Trans. Sig. Process., vol. 60, no. 2, pp. 862-876, Feb. 2012.

[23] P. Rost, "Achievable net-rates in multi-user OFDMA with partial CSI and finite channel coherence," in Proc. of IEEE VTC, pp. 1-5, Sep. 2012.

[24] T. Eriksson and T. Ottosson, "Compression of feedback for adaptive transmission and scheduling," IEEE Proceedings, vol. 95, pp. 2314-2321, Dec. 2007.

[25] H. Gao, R. Song and J. Zhao, "Compression of CQI feedback with compressive sensing in adaptive OFDM systems," in Proc. of IEEE WCSP, pp. 1-4, Oct. 2010 .

[26] C. Xu, L. Zhang, L. Song, Y. Zhao and B. Jiao, "Feedback compression for time-correlated MIMO block-fading channels using huffman coding," in Proc. of IEEE ICCT, pp. 411-415, Sep. 2011. 
[27] B. Makki and T. Ericsson, "Efficient channel quality feedback signaling using transform coding and bit allocation," in Proc. of IEEE VTC, pp. 1-5, May 2010 .

[28] M. Shaqfeh, H. Alnuweiri and M. S. Alouini, "A reduced feedback proportional fair multiuser scheduling scheme," IEEE GLOBECOM Workshops, pp. 14041408, Dec. 2011.

[29] S. H. Hur and B. D. Rao, "Performance of a reduced feedback OFDMA system employing joint scheduling and diversity," in Proc. of IEEE VTC, pp. 1-5, May 2011.

[30] H. Kwon, W. I. Lee and B. G. Lee, "A minimum data-rate guaranteed resource allocation with low signaling overhead in multi-cell OFDMA systems," Journ. of Commun. and Networks, vol. 11, pp. 26-35, Feb. 2009.

[31] J. Gross, H. F. Geerdes, H. Karl and A. Wolisz, "Performance analysis of dynamic OFDMA systems with inband signaling," IEEE J. Select. Areas Commun., vol. 24, pp. 427-436, Mar. 2006.

[32] E. G. Larsson, "Optimal OFDMA downlink scheduling under a control signaling cost constraint," IEEE Trans. Commun., vol. 58, pp. 2776-2781, Sep. 2010 .

[33] J. Gross, H. Karl and A. Wolisz, "On the effect of inband signaling and realistic channel knowledge on dynamic OFDM-FDMA systems," in Proc. of European Wireless 2004, Feb. 2004.

[34] J. Gross, P. Alvarez and A. Wolisz, "The signaling overhead in dynamic OFDMA systems: reduction by exploiting frequency correlation," in Proc. of IEEE ICC, pp. 5143-5148, Jun. 2007.

[35] J. So, "Performance analysis of a semi-fixed mapping scheme for VoIP services in wireless OFDMA systems," in Proc. of IEEE ICWMC, pp. 13-17, Aug. 2009.

[36] O. A. Dobre, A. Abdi, Y. Bar-Ness and Wei Su, "Blind modulation classification: a concept whose time has come," in Proc. of IEEE Symp. on Advances in Wired and Wireless Communication, pp. 223-228, Apr. 2005.

[37] E. Soltanmohammadi and M. Naraghi-Pour, "Blind modulation classification over fading channels using expectation-maximization," IEEE Commun. Letters, vol. 17, pp. 1692-1695, Sep. 2013.

[38] M. Marazin, R. Gautier and G. Burel, "Algebraic method for blind recovery of punctured convolutional encoders from an erroneous bitstream," IET Signal Process., vol. 6, iss. 2, pp. 122-131, Apr. 2012.

[39] M. Cluzeau, "Block code reconstruction using iterative decoding techniques," in Proc. of IEEE Int. Symp. on Information Theory, pp. 2269-2273, Jul. 2006. 
[40] M. Cluzeau and J. P. Tillich, "On the code reverse engineering problem," in Proc. of IEEE Int. Symp. on Information Theory, pp. 634-638, Jul. 2008.

[41] V. Choqueuse, M. Marazin and L. Collin, "Blind recognition of linear spacetime block codes: a likelihood-based approach," IEEE Trans. Signal Process.,, vol. 58, No. 3, pp. 1290-1299, Mar. 2010.

[42] T. Xia and H. C. Wu, "Novel blind identification of LDPC codes using average LLR of syndrome a posteriori probability," in Proc. 12th International Conference on ITS Telecommunications, pp. 12-16, Nov. 2012.

[43] C. Chabot, "Recognition of a code in a noisy environment," in Proc. of IEEE ISIT, pp. 2211-2215, Jun. 2007.

[44] J. Kim and D. H. Cho, "Simultaneous transmission of MAP IE and data for minimizing MAC overhead in the IEEE 802.16e OFDMA systems," IEEE Trans. Wireless Commun., vol. 8, pp. 5431-5435, Nov. 2009.

[45] X. Jin, J. Zhou, J. Hu, J. Shi, Y. Sun and E. Dutkiewicz, "An efficient downlink data mapping algorithm for IEEE 802.16e OFDMA systems," in Proc. of IEEE GLOBECOM, pp. 1-5, Dec. 2008.

[46] H. Jin, J. Y. Lee and D. K. Sung, "On the efficiency of persistent scheduling for non-periodic real-time services in IEEE 802.16e system," in Proc. of IEEE PIMRC, pp. 1481-1486, Sep. 2010.

[47] T. V. K. Chaitanya and E. G. Larsson, "Improving 3GPP-LTE uplink control signaling performance using complex-field coding," IEEE Trans. Veh. Technol., vol. 62, no. 1, pp. 161-171, Jan. 2013.

[48] T. V. K. Chaitanya and E. G. Larsson, "Improving 3GPP-LTE uplink control signaling by repetition across frequency bands," in Proc. of IEEE ICC, pp. 1243-1248, Jun. 2013.

[49] D. Wang, S. Yang, Y. Liao and Y. Liu, "Efficient receiver scheme for LTE PUCCH," IEEE Commun. Letters, vol. 16, no. 3, pp. 352-355, Mar. 2012.

[50] M. R. Raghavendra, S. Nagaraj, K. V. Pradap and P. Flemming, "Robust channel estimation and detection for uplink control signaling in 3GPP LTE," in Proc. of IEEE GLOBECOM, pp. 1-5, Nov. 2009.

[51] Z. Li, Y. Xin and G. B. Giannakis, "Linear constellation precoding for OFDM with maximum multipath diversity and coding gains," IEEE Trans. Commun., vol. 51, pp. 416-427, Mar. 2003. 


\section{Part II}

\section{Transmission of Scheduling Assignments}

The articles associated with this thesis have been removed for copyright reasons. For more details about these see:

http://urn.kb.se/resolve?urn=urn:nbn:se:liu:diva-102537 